\title{
Radiation damage and electron lifetime measurements for liquid ionization chambers
}

\author{
M.J. Longo, L.E. Antonuk, L.H. Oesch and C.F. Wild * \\ Department of Radiation Oncology and Department of Phystcs, University of Michigan, Ann Arbor, MI 48109, USA
}

\begin{abstract}
Ionization chambers filled with liquids at room temperature have begun to be implemented in high-energy physics for total-ionization calorımeters and offer potential applications in medical physics for dosımetry and imaging of radiotherapy beams. One liquid that is commonly used is $2,2,4,4$ tetramethyl pentane (TMP). When suitably purified, the lifetime of electrons in this liquid can be on the order of microseconds, and the mobility is high enough to allow the rapid collection of charge when desirable. In all of these applications, the susceptibility of the liquid to radiation damage is an important question. We present results of studies of radiation damage in TMP and the effect of doses of $\sim 6000 \mathrm{~Gy}$ on the electron lifetime and on the output per unit dose. Our experience in purifying the TMP to optimize the electron lifetime is also described.
\end{abstract}

\section{Introduction}

Ionization chambers filled with liquids at room temperature have begun to be implemented in high-energy physics for total ionization calorimeters [1] and offer potential applications in medical physics for dosimetry [2] and imaging of radiotherapy beams [3]. In conjunction with the development of an imager for radiotherapy, we have investigated the lifetime of electrons in $2,2,4,4$ tetramethyl pentane (TMP) and the susceptibility of this liquid to deterioration due to radiation damage.

\section{TMP purification procedure and test-cell construction}

Highly purified TMP was purchased from Wiley Organics [4] and further purified in the system shown schematically in fig. 1 . The system was constructed completely from stainless steel which was electropolished and checked carefully for leaks with a helium mass spectroscope leak detector. It was flushed with warm ethyl alcohol and HPLC-grade acetone and HPLC water and then with $16 \mathrm{M} \Omega \mathrm{cm}$ deionized water. After bakeout and pumpdown, the system was kept pressurized with research-grade helium. Approximately half of the TMP in the system was boiled off to remove any oxygen or nitrogen dissolved in the liquid. The liquid

\footnotetext{
* Present address: Department of Radiation Oncology, University of Pittsburgh, Presbyterian-University Hospital, 230 Lothrop St., Pittsburgh, PA 15213, USA.
}

was moved by applying helium gas pressure to the appropriate input valve.

The test cell used for the lifetime and radiation damage study is shown in fig. 2. It was constructed of stainless steel with a single ceramic feedthrough and subjected to the same cleaning procedure described above. Connections to the test cell were made with stainless steel connectors and nickel gaskets. After the test cell was connected to the purifier, the connecting tubes could be pumped out so that no impurities were introduced into the system. TMP was circulated through the test cell and filters, which contained a mixture of 5 $\mathrm{A}$ and $13 \mathrm{~A}$ molecular sieve and silica gel, 15 times before the electron lifetime measurements were first made.

\section{Electron lifetime measurements}

The electron lifetime measurements were made with a pulsed beam of bremsstrahlung photons with energies $\sim 6 \mathrm{MeV}$ from a radiotherapy treatment machine. The beam came in bursts approx. $4 \mu \mathrm{s}$ long at $100 \mathrm{~Hz}$. The setup is shown in fig. 3 . High voltage was applied to the cell for $10 \mu \mathrm{s}$ starting at a time $t_{\mathrm{d}}$ after the arruval of the beam burst. Note that under these conditions the transit time of the electrons is very long, $-0.5 \mathrm{~ms}$, so most of the electrons are trapped by impurity atoms to form ions [5]; thus the output vs delay behavior is dominated by the lifetime for electron capture.

The output of the cell was integrated and the average output current was monitored with a digital meter. The variation of the output current with $t_{\mathrm{d}}$ is shown in fig. 4 for TMP before and after the radiation damage studies 


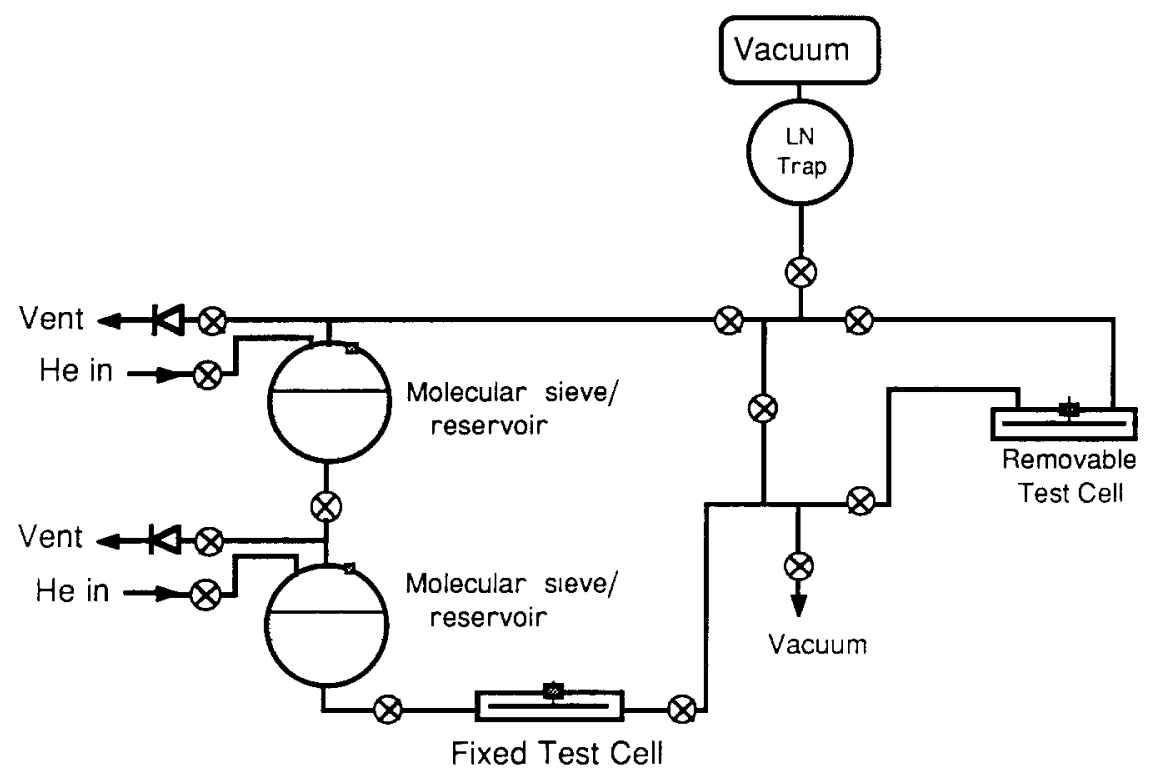

Fig. 1 Purification and fillıng system for the TMP.

discussed below. The exponential slope of this plot gives directly the lifetime of the electrons in the liquid. We obtain a lifetime of approximately $3.1 \mu \mathrm{s}$ before irradiation. Note that this lifetime has not been corrected for the finite width of the beam pulse or of the HV pulse, and so represents an upper limit. A small increase in the electron lifetime to $3.6 \mu$ s was seen after irradiation with $6700 \mathrm{~Gy}$.

Fig. 4 also shows lifetıme data for a chamber approximately $6.0 \mathrm{~cm}$ square with two parallel wire grids spaced $1 \mathrm{~mm}$ apart as electrodes. This chamber was also constructed with stainless steel and ceramic feedthroughs. It was subjected to a purification procedure similar to that described above plus a bakeout at $3000^{\circ} \mathrm{C}$ under vacuum. The final flush with solvents and deionized water was done at CERN by the UAl group. The chamber was filled with TMP purified as described in Albrow et al. [1], then sealed and returned

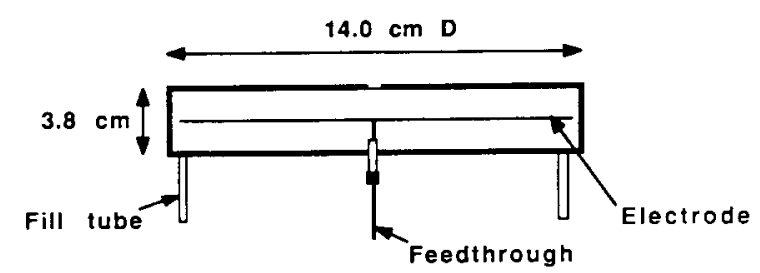

Fig. 2. Test cell used in the lifetıme and radiation damage measurements. The chamber was made entirely of stainless steel except for the ceramic feedthrough. to us. The electron lifetime for this chamber is approx. $3.2 \mu \mathrm{s}$ from the data in fig. 4 .

\section{Radiation damage studies}

The radiation damage studies were made by exposing the cell to a $2300 \mathrm{Ci}{ }^{60} \mathrm{Co}$ source with the setup in fig. 5. The dc output of the cell was continuously monitored during 1rradiation. The output of the cell is plotted vs time and integrated dose in fig. 6 . Over the first $120 \mathrm{~min}$ of the irradiation the output of the cell increased approximately $50 \%$. Over the next $72 \mathrm{~h}$ the output slowly decreased by approximately $7 \%$. The discontinuities near $t=160,780$ and $1650 \mathrm{~min}$ occur when the exposure was interrupted for periods of several days

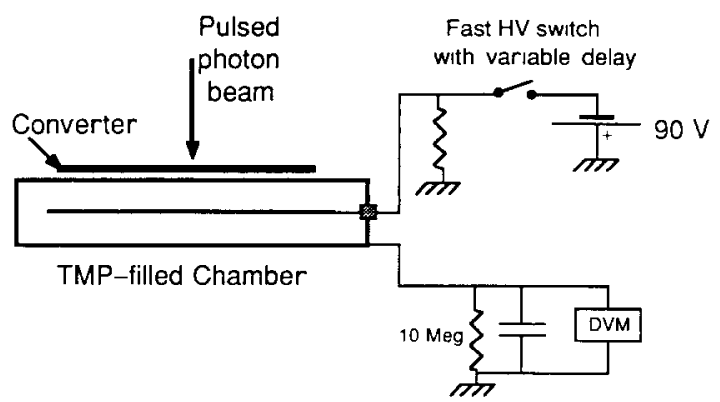

F1g. 3. Arrangement for the electron lifetime measurements. The HV switch was an optocoupler which was switched on for $10 \mu \mathrm{s}$ at a variable time delay relative to the beam arrival time. 


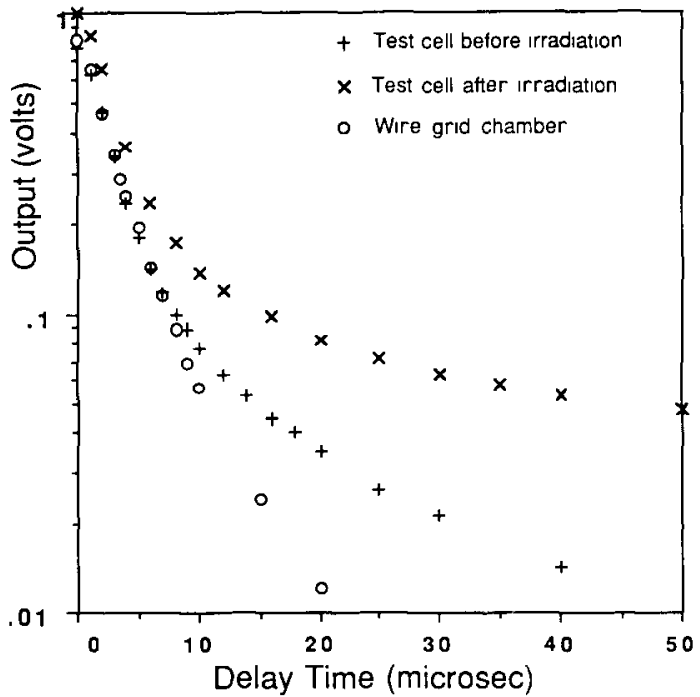

Fig. 4. Output of the test cell vs. delay of the HV pulse, before and after irradiation with $6700 \mathrm{~Gy}$. Data for another cell with wire grid electrodes, which was filled at CERN, are also shown.

or more. After each interruption the output was found to start off slightly lower, then increase over a period of $30 \mathrm{~min}$, then decrease to approximately the value observed before the interruption. These variations were $\sim 10 \%$.

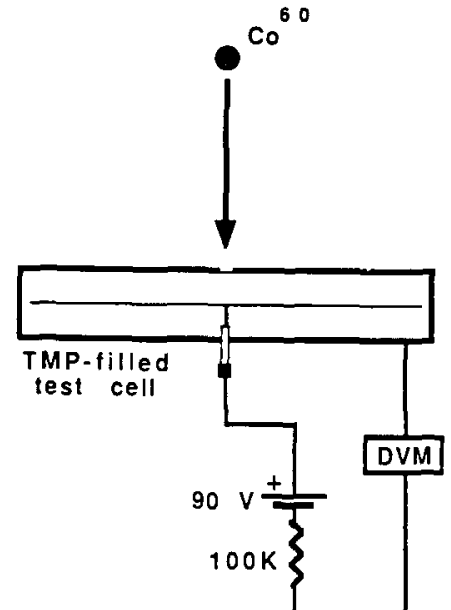

Fig. 5. Setup for the radiation damage studies.

\section{Discussion}

The lifetimes for electrons in TMP observed in this experiment are considerably shorter than the $>100 \mu \mathrm{s}$ lifetimes reported by Radermacher [6] and the $77 \mu \mathrm{s}$ lifetime reported by Ochsenbein et al. [7]. On the other hand, Aubert et al. [8] report a lifetime of $2.47 \pm 0.18 \mu \mathrm{s}$ which is comparable with our value. We note that Radermacher et al. and Ochsenbein et al. used an indirect method to infer the lifetıme from the pulse shape observed for muons traversing the chamber, while Aubert et al. used a pulsed-neutron-beam method. The

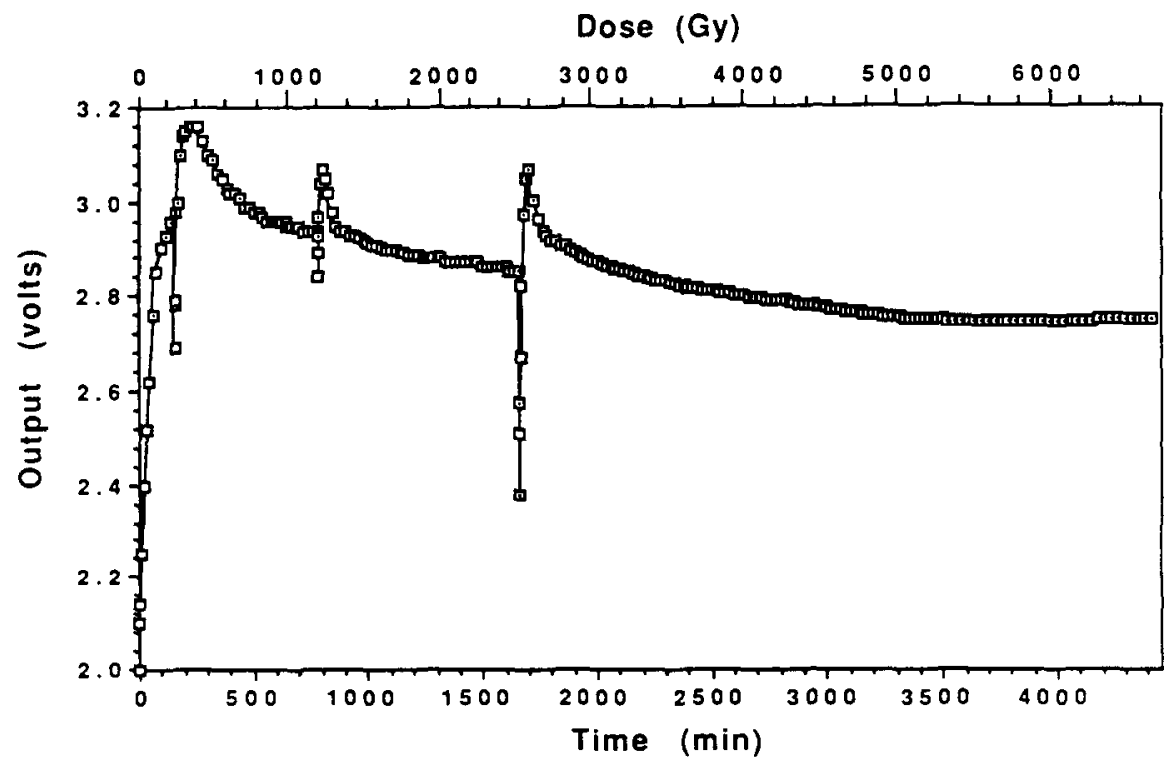

Fig. 6. Output of the test cell during irradiation vs. exposure tıme and radiation dose. Note the suppressed zero on the vertical scale. 
method used for our measurements is more direct, as the variation of the output of the chamber with increasing delay between the ionization pulse and the application of the high voltage is observed.

The behavior of the output of the cell with continued irradiation shown in fig. 6 is obviously quite complicated. The initial rise in output is probably due to the trapping of impurity ions on the stainless-steel electrodes which leads to a modest increase in electron lifetime and thus cell output. When the irradiation is interrupted, some of the impurities return to the liquid so there is a temporary decrease in output. These variations would make it difficult to use this type of cell in radiation dosimetry and could cause stability problems in total absorption calorimetry. Our earlier studies with less pure TMP showed much larger variations with accumulated dose. This suggests that these effects will dıminısh with increasing purity.

On the other hand, the overall response shows only a small decrease in output with doses of $6700 \mathrm{~Gy}$. The lifetıme data in fig. 4 are reasonably well fitted to the sum of two exponentials. The behavior for the TMP before irradiation shows a dominant component with a lifetime of approximately $3.1 \mu \mathrm{s}$ and a component $10 \%$ with a lifetime $\tau \sim 40 \mu \mathrm{s}$. After irradiation, the lifetime had increased to about $3.6 \mu \mathrm{s}$ for the shorterlived component and to $\sim 125 \mu \mathrm{s}$ for the longer-lived. The longer-lived component may be due to ions.

\section{Acknowledgements}

We wish to thank E. Radermacher and other members of the UAl group at CERN for advice on chamber construction and for filling a test chamber. The efforts of J. Boudry, I. Yorkston and T. Azemoon who contributed to various aspects of this work are gratefully acknowledged.

\section{References}

[1] See, for example, J. Engler, H Keım and B Wild, Nucl Instr. and Meth. A252 (1986) 29; M. Albrow et al., Nucl. Instr and Meth. A265 (1988) 303

[2] See, for example, G. Wickman, Acta Radiol. Therapy Phys. Biol. 13 (1974) 37.

[3] M. Van Herk and H Meertens, Radiotherapy and Oncology 11 (1988) 369.

[4] Wiley Organics, 1245 South 6th St., Coshocton, OH 43812 , USA

[5] Recombination of the electron with the positive ion from which it originated is small, even at $E=0$. See R.A Holroyd and D F Anderson, Nucl. Instr. and Meth. A236 (294) 1985

[6] E Radermacher, CERN-EP/89-01 (Jan 1989) unpublished.

[7] S. Ochseṇbein et al. Nucl. Instr. and Meth. A273 (1988) 654

[8] B Aubert et al., Nucl. Instr. and Meth. A279 (1989) 126. 